\title{
Mass Integration for Rigorous Process Modelling: A Sequential Approach for Direct Recycling
}

\author{
Filippo Marchione ${ }^{1}$, Konrad Hungerbuehler ${ }^{1}$ and Stavros Papadokonstantakis ${ }^{2 *}$ \\ ${ }^{1}$ ETHZ, Department of Chemistry and Applied Biosciences, Zurich, Switzerland, ${ }^{2} T U$ Wien, Institute of Chemical, Environmental \\ and Bioscience Engineering, Vienna, Austria
}

OPEN ACCESS

Edited by:

Seyed Reza Nabavi,

University of Mazandaran, Iran

Reviewed by: Juan Gabriel Segovia Hernandez, University of Guanajuato, Mexico

Ali Farzi,

University of Tabriz, Iran

*Correspondence:

Stavros Papadokonstantakis stavros.papadokonstantakis@ tuwien.ac.at

Specialty section:

This article was submitted to Computational Methods in Chemical Engineering,

a section of the journal

Frontiers in Chemical Engineering

Received: 28 September 2021 Accepted: 03 November 2021

Published: 01 December 2021

Citation:

Marchione F, Hungerbuehler $K$ and Papadokonstantakis S (2021) Mass Integration for Rigorous Process Modelling: A Sequential Approach for

Direct Recycling.

Front. Chem. Eng. 3:785179,

doi: 10.3389/fceng.2021.785179
Mass integration has been used for reducing the amount of process waste and environmental impact. Despite its long history, new challenges constantly arise with the use of process simulation tools offering platforms for rigorous process models. Therefore, the typical mass integration framework requires modifications to accurately account for the process performance. In this work, a novel sequential methodology is presented to realize a recycle network with rigorous process models. Initially, under the hypothesis of constant compositions of the process sources, an optimal ranking of the process sinks is determined. The optimal recycling network thus obtained is then used for a sequential methodology considering rigorous process models. The violations of process constraints are handled at each sequential step through the concept of "tightening constant". The application of the sequential methodology to two case studies proves its ability to provide good approximations of the global optima with low computational effort.

Keywords: process integration, process simulation, preemptive goal programming, nonlinear optimization, Aspen Plus simulation

\section{INTRODUCTION}

In the wider set of optimization techniques applied to chemical engineering, process integration has proven to be a very useful tool to enhance the profitability of industrial processes and reduce their environmental footprint, through the reduction of raw materials, waste discharge and energy consumption. Among the various approaches of process integration (e.g., direct recycling, energy integration, property integration, mass exchange networks) mass integration through direct recycling and mass exchange networks allow to recover part of the waste streams of a process and reuse them directly or after purification, thus reducing the purchase of pure raw

\begin{abstract}
Abbreviations: $b$, tightening constant; $\mathrm{DI}_{\mathrm{RPM}}$, degree of integration with rigorous process modelling; DS, process design specification; $i, j$, indices for generic sinks and sources; $k$, kinetic constant of a reaction; MAI, maximum allowable impurity of a target compound; $\mathrm{MAI}_{\mathrm{SK}}$, maximum allowable impurity of a target compound for a process sink $\mathrm{SK}$; $\mathrm{MAI}_{\text {tight }}$, tightened maximum allowable impurity; NPM, problem representation without rigorous process modelling; OSK $_{\mathrm{NPM}}$, ordered process sinks obtained without rigorous process modelling; $\mathrm{OSK}_{\mathrm{RPM}}$, ordered process sinks obtained with rigorous process modelling; PC, process constraint; PM, process model; R, ideal gas constant $\left(8.314 \mathrm{~J} \mathrm{~mol}^{-1} \mathrm{~K}^{-1}\right)$; $\mathrm{RN}_{\mathrm{NPM}}$, recycling network obtained without rigorous process modelling; $\mathrm{RT}_{\mathrm{NPM}}$, recycling target obtained without rigorous process modelling; $\mathrm{RN}_{\mathrm{RPM}}$, recycling network obtained with rigorous process modelling; $\mathrm{RPM}$, rigorous process modelling; $\mathrm{RT}_{\mathrm{RPM}}$, recycling target obtained with rigorous process modelling; SK, process sink; SMI, sequential mass integration; $\mathrm{SMI}_{\mathrm{NPM}}$, sequential mass integration without rigorous process modelling; $\mathrm{SMI}_{\mathrm{RPM}}$, sequential mass integration with rigorous process modelling; $\mathrm{SQP}$, successive quadratic programming; SR, process source.
\end{abstract}


materials and the generation of waste (El-Halwagi, 2006; ElHalwagi \& Manousiouthakis, 1989, 1990).

Many past works proposed graphical approaches (Wang \& Smith, 1994; Hallale, 2002; El-Halwagi et al., 2003; El-Halwagi, 2006) and algebraic methods (Sorin \& Bedard, 1999; Manan et al., 2004; El-Halwagi, 2006; Foo et al., 2006) to calculate the target for minimum fresh resource and maximum recycling. These works mainly focus on continuous, steady state processes, which lie in the background, while the recycling network and its respective maximum recycling target lie in the foreground of the analysis. Interactions between the foreground and the background processes are mainly expressed through the contained impurities in the process sources (i.e., waste streams), the concept of maximum allowable impurity in the process sinks (i.e., process units receiving the recycling streams) and sometimes simplified effects in the form of linear or nonlinear expression between impurities and process related parameters.

Process integration techniques have also been extended to batch processes (Majozi, 2005; Majozi et al., 2006; Gonws et al., 2010; Adekola \& Majozi, 2011). Similar to the case of continuous processes, not much attention has been paid on the interaction of the impurities with the dynamic behaviour of the process unit operations. Typically, the process unit operation models are significantly simplified and more attention is paid at problems of higher level of abstraction, such as the interaction of mass integration approaches with scheduling and planning issues.

Recently, the mass integration problems have extended their scope toward more complicated superstructures and problem formulations, dealing in particular with the significant algorithmic challenges for computationally efficient solution. In this direction, the mathematical formulation of Seid and Majozi (2014) aims at improving the profitability of multipurpose batch plants, minimizing simultaneously wastewater generation and utility usage. Furthermore, superstructures for the synthesis of heat-integrated water networks, considering also wastewater treatment units have been investigated (Ahmetovic \& Kravanja, 2013; Ahmetovic et al., 2014; Ibric et al., 2014). Gabriel and El-Halwagi (2005) proposed an approach to reformulate the problem of synthesizing the interception and direct recycle and reuse of process waste into a linear problem, thus simplifying the calculation of the global optimum. Alva-Argaez et al. (2007) and Faria and Bagajewicz (2008) worked on water network superstructures expressing the principal design problem in terms of mixed-integer-nonlinearprogramming (MINLP) which is subsequently decomposed into mixed-integer-linear-programming (MILP) to be efficiently solved. Kheireddine et al. (2011) afforded mass and property integration, considering simultaneously thermal constraints and Jimenez-Gutierrez et al. (2014) formulated an MINLP model considering simultaneously energy, mass and property integration for the synthesis of water networks. Another type of extension of the mass integration scope refers to those works taking account of multiple contaminants in process streams, for instance for the optimization of wastewater networks (Boix et al., 2011; Tudor \& Lavric, 2011; Napoles-Rivera et al., 2012).

Sequential approaches applied to process integration problems have also been proposed at a higher level of abstraction. For instance, Halim and Srinivasan (2009) presented a framework for optimizing the profitability of batch plants where the scheduling problem and the heat-integration problem are solved successively; the same authors extended their approach to successively calculate optimal economic, energy and water reuse targets for alternate schedules (Halim and Srinivasan, 2011).

To the best of our knowledge, very limited research effort has been dedicated to afford mass integration under rigorous process modelling conditions. For instance, in the topic of water integration and synthesis of water networks, which covers a significant part of the mass integration framework in previously published literature, the impact of recycled contaminants over the performance of process units is analyzed under very specific conditions. Wang and Smith (1994) distinguish the regeneration units between fixed outlet contaminant composition and fixed removal ratios, Karuppiah and Grossmann (2006) consider fixed contaminant production in the process units, and Ahmetovic and Grossmann (2011) include a rather simple process model in the form of stoichiometric relationships in the water regeneration units. Still, even in this case, the impact of recycled contaminants to the background process producing the water effluents is not studied, since direct recycling (i.e., with no water purification units) has not been combined with rigorous background process models.

From a first aspect, a problem formulation of this kind typically requires significant computational effort; thus, it is not a straightforward extension of the existing MINLP approaches and is typically handled with simplifying assumptions regarding the utilized process models. Another more practical issue is that specialized software would be required, which can simultaneously handle detailed process modelling (i.e., describing in full extent the nonlinear relations of thermodynamic properties and equilibrium, chemical reaction kinetics etc.) and an advanced optimization platform. Some efforts in this direction consider superstructure development, simulation and optimization in Aspen Plus (Malik et al., 2015), nonlinear-linear programming (NLP) for planning and optimization with rigorous process models (Alhajri et al., 2008) and rigorous flowsheet optimization using process simulators (Caballero \& Grossmann, 2008). Recent works (Handani et al., 2015; Porzio et al., 2016) afforded mass integration with process models based on the use of stoichiometric and equilibrium model reactors, process simulation and optimization.

This study aims at providing new insights in the problem of mass integration coupled with rigorous process modelling, on the basis of a novel sequential approach to realize a direct recycling network. The rigor of the model consists in typical equations of thermodynamic properties and equilibria, reaction kinetics, stream mixing and heat exchange, such as those included in commercial process simulators (e.g., Aspen Plus). The novel sequential approach consists of two parts. First, an optimal order of process units (i.e., process sinks) for integration is obtained by neglecting the rigor of the process models and solving a succession of linear programming problems. This optimal order of process sinks is subsequently used to realize a 
direct recycling integration network step by step by considering the rigorous process models. More than one version of this algorithmic approach is discussed for the sake of optimality and in the expense of algorithmic simplicity.

This approach is of particular interest when simplifications in process modelling performance are expected to have a larger impact in the optimised solution compared to the rigor of a global optimisation approach using simplified process models. Moreover, the interaction of the decision maker with the sequential integration approach can be readily facilitated because of the possibility of a step by step interpretation of the process modelling results.

The novel sequential methodology for direct recycling has been applied to two case studies. The first one represents a problem of real industrial complexity, where process information from a non-integrated industrial plant for fine chemical production has been used to construct a detailed process model in Aspen Plus ${ }^{\circledR} 8.6$ (www.aspentech.com). Despite the process complexity, it is still possible in this case, although computationally expensive, to calculate the global optimum of the direct recycling problem. This is then used to assess the performance of the novel sequential methodology regarding its optimality gap. At the same time, the advantages of the novel method in terms of interpretability are clearly demonstrated. The second case study represents a generic process, which is constructed with the purpose to highlight and test the sequential methodology for some particular problematic cases that may appear in mass integration under rigorous process modelling conditions. Finally, this work concludes with suggestions for further investigation of algorithmic parameters of the sequential methodology to reduce the optimality gap and discusses the respective computational trade-offs.

\section{METHODOLOGY}

In this section, an algorithm based on linear optimization problems is first outlined. It allows to optimally order the process sinks in case of mass integration through direct recycling under the assumption of unaffected stream compositions after closing the recycling loops. The details of this approach have been presented elsewhere (Marchione et al., 2016).

Subsequently, the obtained order of process sinks will be used as the basis for the sequential methodology to realize a recycle network coupled with rigorous process models. The rigorous process models capture the impact of the recycled impurity on the output streams of the process sink where it is recycled and the propagation in the process flowsheet up to the process sources (i.e., the origin of the recycling loop). This has the consequence that the previously derived recycling targets of the optimal order of the process sinks have to be properly adjusted to satisfy the maximum allowable impurity and other process constraints. For this reason, a tightening and relaxing algorithmic approach is introduced and this concept is demonstrated in a simple example.

\section{Sequential Mass Integration Without Rigorous Process Modelling (SMI NPM $_{\text {) }}$}

In the framework of mass integration through direct recycling, a set of process units, called process sinks, receive as input streams a certain quantity of a pure compound, referred to as target compound. Such input streams, also called fresh sources, are supposed to be partially or totally replaced by a set of process waste streams available for recycling, called process sources.

All process sources contain the target compound together with other substances, considered in general terms as impurity. Each process sink is characterized by a maximum allowable impurity constraint, which refers to the impurity fraction in the mixed stream entering the process sink.

In the absence of rigorous process modelling, the problem of maximizing the recycling of the target compound from the process sources to the process sinks must be based on the simplifying assumption that the recycled impurities have a negligible impact on the output variables of the process sinks. Consequently, the compositions of all the process sources are also not affected by the recycling loops (i.e., since there is no impact of the recycling to the process sinks, there is also no impact to propagate through the flowsheet streams up to the process sources). Vice versa, when such an assumption is a priori made, there is no need to include any process model, at least as far as the mass integration through direct recycling is concerned.

Under this simplifying assumption, the respective optimization problem (i.e., the maximization of the target compound recycling) can be solved using linear programming (LP) (El-Halwagi, 2006); the solution also corresponds to the minimum quantity of fresh source of the target compound needed by the process sinks. It has been also demonstrated (Marchione et al., 2016) that it is possible to reach the maximum target for recycling in a sequential mass integration approach $\left(\mathrm{SMI}_{\mathrm{NPM}}\right)$, while simultaneously ordering the process sinks in terms of recycled amount (i.e., constructing the recycling network starting from the process sink that can accept the maximum amount of recycling and ending with the process sink that accepts the minimum amount of recycling). This is achieved by following a linear preemptive goal programming approach (Baykasoglu, 2005), which is illustrated in Figure 1 and shortly outlined in the following.

From the set of available sources and sinks, the process unit with the highest recycled amount is identified (primary objective), respecting the constraints of maximum process sink load and allowable impurity. This amount is then fixed as a new constraint in the problem while a sub-algorithm is executed to maximize the amount of impurity recycled to this process sink (secondary objective). This sub-algorithm also minimizes the recycled amount from the purest process sources in succession (further objectives), based on their impurity fraction. This is a procedure of successive linear programming problems, where the solution of each problem becomes an additional constraint for the next problem.

At the end of each process sink selection step, the sets of available process sources and sinks are updated, excluding the 


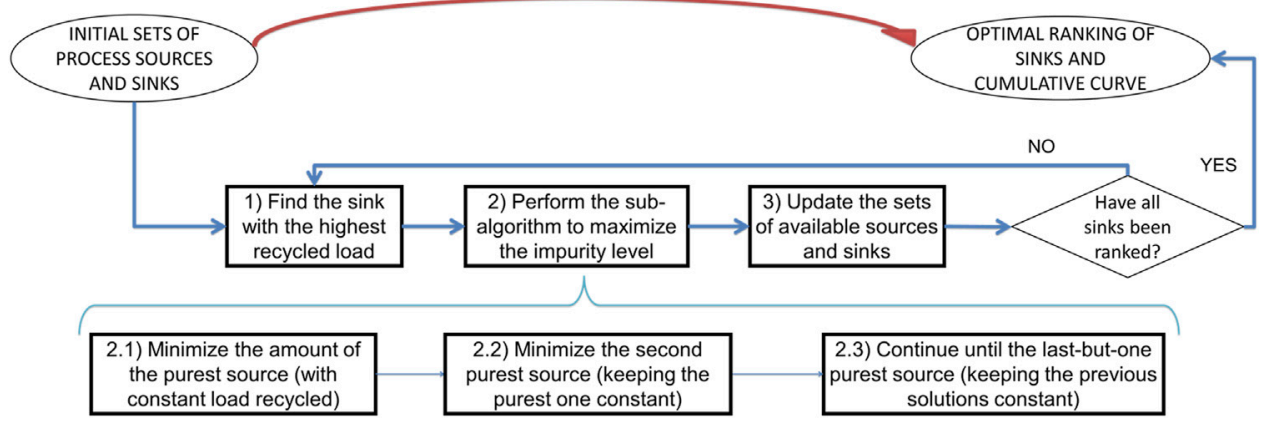

FIGURE 1 | Flowchart describing the sequential mass integration approach without rigorous. process modelling (SMINPM): out of an initial set of process sources and sinks an optimum order of process sinks (OSK $\left.K_{N P M}\right)$, the respective cumulative curve up to the total recycling target (RT $\left.T_{N P M}\right)$ and the recycling network (RN NPM $)$ are calculated by preemptive goal programming on the basis of a succession of linear programming problems.

fractions of process sources which have already been recycled and the process sinks which have already been selected (i.e., ordered). The preemptive goal programming approach is repeated with these reduced sets of process sources and sinks to identify the next process sink of the optimal series. Following this procedure, the conditionally maximum recycled amount is obtained at each process sink selection step, meaning that this recycled amount is the optimal one if the recycling network to the previously selected process sinks is fixed. Thus, it is possible to build a cumulative recycling curve, which is a polygonal curve where each vertex corresponds to the conditionally maximum recycled amount for the subset of ordered process sinks in the respective algorithmic step. The final point of the curve is the maximum recycled amount of the target compound for the whole set of process sinks and is equal to the solution found by the typical superstructure problem formulation using LP. It is important to note that each segment of the polygonal curve has a higher (or equal) slope than the next one.

\section{Extension of the Sequential Mass Integration for Rigorous Process Modelling (SMI RPM}

When rigorous process modelling is used, it is possible to evaluate more precisely how the process units and streams are affected when part of fresh sources is replaced by recycled amounts of the target compound existing in impure waste streams. The term "rigorous process models" refers to sets of linear and nonlinear relations including, for instance, reaction kinetics, thermodynamic properties and equilibria and, more generally, all kinds of input-output relations regarding the process units and streams. In this case, the recycling loops may lead to a change of the compositions of the output streams from the respective process sink, which can propagate through the process flowsheet. This can have two types of impacts: change of the amounts and compositions of the process sources, which may result in violation of the maximum allowable impurity constraints, and violation of other process design specifications. Clearly, both types of impacts have a negative effect on the validity of the $\mathrm{SMI}_{\mathrm{NPM}}$ results. Thus, a procedure is required to optimally adjust the $\mathrm{SMI}_{\mathrm{NPM}}$ results under rigorous process modelling conditions. This procedure is called $\mathrm{SMI}_{\mathrm{RPM}}$ in the rest of the paper and is outlined in Figures 2A,B.

In particular, Figure $\mathbf{2 A}$ presents the information flow connecting $\mathrm{SMI}_{\mathrm{NPM}}$ and $\mathrm{SMI}_{\mathrm{RPM}}$. The two approaches share a common problem formulation in terms of process sinks, sources and constraints referring to maximum allowable impurity (MAI) for each process sink, while the process model and other type of process constraints are only used in $\mathrm{SMI}_{\mathrm{RPM}}$. The results provided by the $\mathrm{SMI}_{\mathrm{NPM}}$ (i.e., maximum recycling target $\left(\mathrm{RT}_{\mathrm{NPM}}\right)$, recycling network $\left(\mathrm{RN}_{\mathrm{NPM}}\right)$ and optimal order of process sinks $\left(\mathrm{OSK}_{\mathrm{NPM}}\right)$ ) are used in the $\mathrm{SMI}_{\mathrm{RPM}}$ to realize a recycling network applying the rigorous process models. In particular, the structure of the recycling network and the order of process sinks are strictly followed in a nonlinear preemptive goal programming approach (Figure 2B), while the $\mathrm{RT}_{\mathrm{NPM}}$ is used as a first approximation of the respective recycling target for the rigorous process model $\left(\mathrm{RT}_{\mathrm{RPM}}\right)$. This means that $\mathrm{RN}_{\mathrm{NPM}}=\mathrm{RN}_{\mathrm{RPM}}$ and $\mathrm{OSK}_{\mathrm{NPM}}=$ OSK $K_{R P M}$, while a degree of integration can be defined $\left(\mathrm{DI}_{\mathrm{RPM}}\right)$ expressing the relative difference between $\mathrm{RT}_{\mathrm{NPM}}$ and $\mathrm{RT}_{\mathrm{RPM}}$.

As shown in Figure $\mathbf{2 B}$, the nonlinear preemptive goal programming approach comprises two different methods. According to the one method (i.e., sequential optimization) for each selected process sink $\left(\mathrm{SK}_{\mathrm{i}}\right)$ only one recycling flow is adjusted at a time to deal with the discrepancies in the process constraints when applying the $\mathrm{RN}_{\mathrm{i}}$ up to the $\mathrm{RT}_{\mathrm{i}}$ of the $\mathrm{SMI}_{\mathrm{NPM}}$ in the rigorous process model. The alternative method (i.e., simultaneous optimization) adjusts at once all source-tosink recycling streams for $\mathrm{SK}_{\mathrm{i}}$ using the rigorous process model. The two methods are explained in more detail in Sequential Source-To-Sink Optimization and Simultaneous Source-to-Sink Optimization.

\section{Sequential Source-to-Sink Optimization}

As shown in Figure 2B, at a generic process sink selection step $\left(\mathrm{SK}_{\mathrm{i}}\right)$, the respective $R \mathrm{~N}_{i}$ is realized up to the $R T_{i}$ value of the $\mathrm{SMI}_{\mathrm{NPM}}$ in the rigorous process model. Because of the rigorous process modelling conditions, this may cause diverse violations of process constraints, including also those referring to the MAI of the $\mathrm{SK}_{\mathrm{i}}$. In case of such process constraint violations, those 


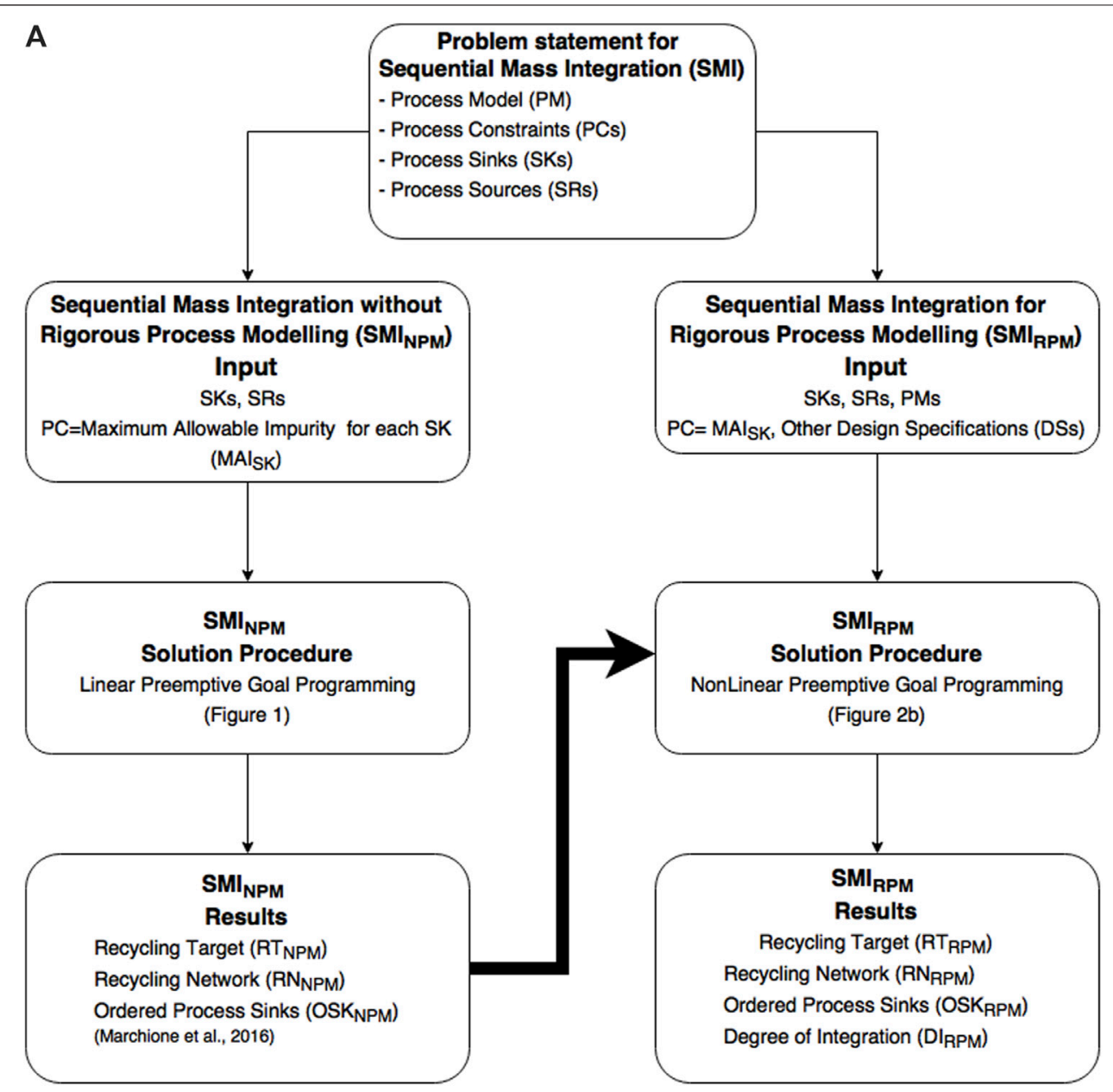

FIGURE 2 | Flowchart describing the sequential mass integration approach with rigorous process modelling (SMI RPM): (A) Overview of the sequential mass

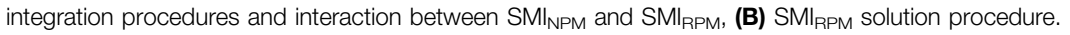

process sources recycling to the $\mathrm{SK}_{\mathrm{i}}\left(\mathrm{SR}_{\mathrm{ij}}\right)$ are ordered according to their actual impurity concentration in a descending order. The first process source of this order (i.e., the most impure one) is then maximized while satisfying all process constraints. This is a rather simple single-variable nonlinear programming problem (NLP). The most impure process source is selected to fix the process constraint violations because it is assumed that this source will have the maximum impact on the selected process sink and thus it will be the one that requires the minimum change of its respective value according to the $\mathrm{SMI}_{\mathrm{NPM}}$ solution. If, however, no recycling value of this process source can be found (i.e., $\mathrm{SR}_{\mathrm{ij}}=0$ ), the next source of the descending impurity order is maximized, until one process source with $\mathrm{SR}_{\mathrm{ij}} \neq 0$ is found.

In the case of no process constraint violations, the process sources are ordered according to their actual impurity concentration in an ascending order. Starting from the purest source, the recycled amount is maximized, while satisfying all process constraints; only if the recycled amount of this process source is exhausted, the next process source of the order is utilized, otherwise the algorithmic procedure is terminated for this process sink selection step.

The procedure is then repeated for all the process sinks according to the $\mathrm{OSK}_{\mathrm{NPM}}$. Following this order of process sinks, it is practically assumed that the bigger recycling amounts have also the greatest impact on the process performance.

For any given process source ordered according to the impurity concentration criteria described above, this single variable optimization procedure can be formulated in Eqs. 1-10:

$$
\max w_{i, n}^{r e c}
$$

s.t.:

$$
\begin{gathered}
w_{k, n}^{r e c}=0 \quad \forall k(0<\mathrm{k}<\mathrm{i}) \\
w_{k, n}^{r e c}=\text { const }_{k} \forall k(\mathrm{i}<\mathrm{k}<\mathrm{NS}) \\
0 \leq z_{j} \leq M A I_{j}, \quad \forall j(1 \leq \mathrm{j} \leq \mathrm{NSK}, \mathrm{j} \neq \mathrm{n})
\end{gathered}
$$




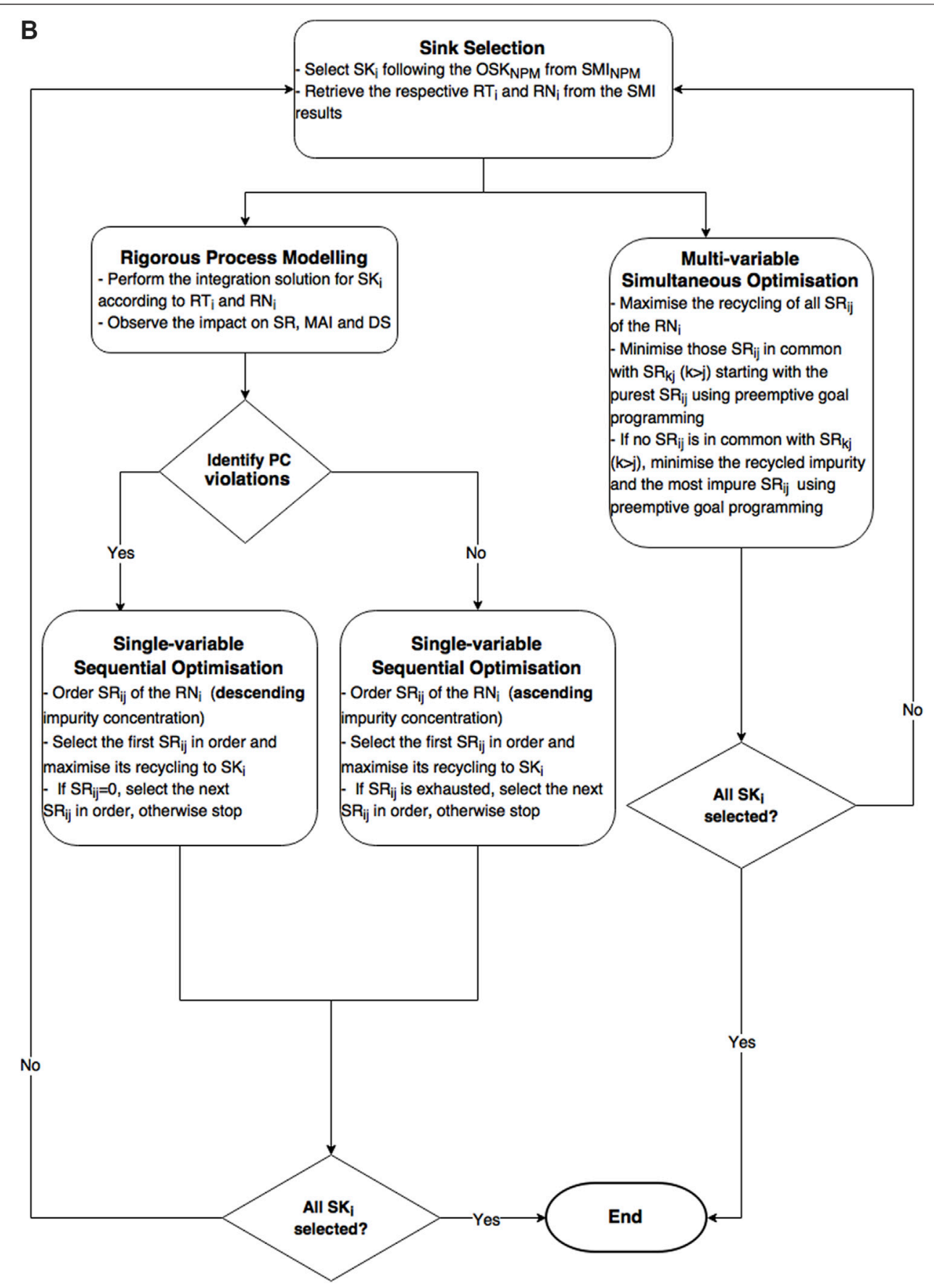

FIGURE 2 | continued.

$$
\begin{gathered}
0 \leq z_{n} \leq M A I_{n, \text { tight }}=(1-b) M A I_{n} \\
g_{n}=\sum_{i=1}^{N S} w_{i, n}^{r e c}+f_{n} \\
g_{n} z_{n}=\sum_{i=1}^{N S} w_{i, n}^{r e c} y_{i} \\
F m(p a r)=0 \\
f_{n} \geq 0
\end{gathered}
$$

$$
w_{i, n}^{r e c} \geq 0
$$

where NSK is the total number of process sinks, and NS is the number of the sources connected to the sink $n$ as a result of the procedure presented in Figure 1 (linear PGP). For any given $i$ process source in order, the recycled amount $\left(w_{i, n}^{r e c}\right)$ is maximized while all the other $\left(w_{k, n}^{r e c}, k>1\right)$ are kept constant $\left(\right.$ const $\left._{k}\right)$ at the 
value previously determined by the PGP (i.e., Figure 1) or are null $\left(w_{k, n}^{r e c}, k<1\right)$, because this would be the only reason for considering another source in the order. In other words, whenever a process source is maximized at a value different than zero, the procedure is stopped and the next sink is considered. In this problem formulation, par is a vector of process variables (e.g., flowrates, compositions, temperatures, pressures), and $\mathrm{Fm}$ denotes a set of equations expressing the process model (e.g., mass and energy balances, reaction rates, thermodynamic equilibria, design specifications). The tightening constant $b$ is a parametric input to the problem (e.g., determined by sensitivity analysis). This concept is illustrated in more details later in the text (paragraph 2.2.3) and is used to reduce the degree of integration in earlier steps of the sequential approach and increase the potential in later steps. Tightening. When $\mathrm{b}=0$, Eq. 5 is redundant. As indicated by Eqs. 4, 5, b can take a non-zero value only for the specific process sink $n$ under investigation and is set back to 0 when the next process sink is considered.

\section{Simultaneous Source-To-Sink Optimization}

As shown in Figure 2B, at a generic process sink selection step $\left(\mathrm{SK}_{\mathrm{i}}\right)$, the respective $\mathrm{RN}_{\mathrm{i}}$ is considered for the simultaneous maximization of the total recycling amount from all the relevant sources $\left(\mathrm{SR}_{\mathrm{ij}}\right)$. This optimization is realized under rigorous process modelling conditions and is thus a multivariable NLP. Still, it is of considerably smaller size compared to solving the NLP for the whole superstructure.

Subsequently, those $\mathrm{SR}_{\mathrm{ij}}$ are identified which are common with the sources used in at least one following process sink selection step. The common $\mathrm{SR}_{\mathrm{ij}}$ are ordered according to their impurity concentration in an ascending order. Then, the purest source from this order is minimized, while keeping constant the maximum recycling amount found previously by the NLP. The next sources of the series are then minimized, one at a time, in a preemptive goal programming approach, namely keeping each time all the previous solutions constant. The reason for utilizing this type of ordering of the process sources is based on the empirical evidence that minimizing the purest process sources in succession implies their maximum saving for the next process sink selection steps, so as to exploit them as much as possible.

The proposed approach is formulated for the case of any given ordered sink in terms of a lexicographic optimization approach, as shown in Eqs. 11-19:

$$
\begin{gathered}
\max _{l e x}\left(\sum_{i=1}^{N S} w_{i, n}^{r e c},-w_{1, n}^{r e c},-w_{2, n}^{r e c}, \ldots,\right. \\
\left.-w_{N C S, n}^{r e c}\right), \quad i \in(1,2, \ldots, N S)
\end{gathered}
$$

s.t.

$$
\begin{gathered}
w_{i}=\sum_{i=1}^{N S} w_{i}^{r e c}+w_{i}^{\text {waste }} \forall i \in(1, \ldots, \mathrm{NS}) \\
0 \leq z_{j} \leq M A I_{j} \forall j \in(1 \leq \mathrm{j} \leq \mathrm{NSK}, \mathrm{j} \neq \mathrm{n}) \\
0 \leq z_{n} \leq M A I_{n, \text { tight }}=(1-b) M A I_{n}
\end{gathered}
$$

$$
\begin{gathered}
g_{n}=\sum_{i=1}^{N S} w_{i, n}^{r e c}+f_{n} \\
g_{n} z_{n}=\sum_{i=1}^{N S} w_{i, n}^{r e c} y_{i} \\
F m(p a r)=0 \\
f_{n} \geq 0 \\
w_{i, n}^{r e c} \geq 0, \quad \forall i \in(1, \ldots, \mathrm{NS})
\end{gathered}
$$

where NCS is the number of common sources between the given process sink and the next process sinks in order.

If there are no common $\mathrm{SR}_{\mathrm{ij}}$ with the next steps, the process sources are ordered according to their impurity concentration in a descending order. The first source of this order (i.e., the most impure source) is minimized while keeping constant the maximum recycled load found previously by the NLP. The next sources of the series are then minimized, one at a time, in a preemptive goal programming approach, namely keeping each time all the previous solutions constant. The reason for utilizing this type of ordering of the process sources is based on the empirical evidence that minimizing the most impure process sources in succession leads to the minimum quantity of recycled impurity, and thus to the minimum impact on the process performance. The procedure is then repeated for all the process sinks according to the $\mathrm{OSK}_{\mathrm{NPM}}$.

\section{Sequential Tightening and Relaxing of the Maximum Allowable Impurity Constraints}

The total recycled loads provided by the two aforementioned methods can be further increased based on the empirical observation that reducing slightly the degree of integration at a generic process sink selection step may significantly improve the recycled amounts in the following steps. One way of reducing the degree of integration at a generic process sink selection step consists in decreasing, or tightening, by a factor $b$ (\%) the maximum allowable impurity of process sinks, as shown in Eq. 20:

$$
M A I_{\text {tight }}=(100-\mathrm{b}) \cdot M A I
$$

The factor $b$ is defined as "tightening constant". This tightening of the MAI constraint is only valid at the present process sink selection step and is relaxed back to its original value at the next process sink selection steps. It is expected that the use of a tightening constant $b$ up to a certain point can improve the $\mathrm{RT}_{\mathrm{RPM}}$. Indeed, high values of $b$ are expected to be too restrictive for improving the $\mathrm{RT}_{\mathrm{RPM}}$. An optimal tightening constant that maximizes $\mathrm{RT}_{\mathrm{RPM}}$ can be found by means of sensitivity analysis.

The concept of tightening and relaxing the MAI constraints is illustrated in a simple example for the process depicted in Figure 3.

Compound $\mathrm{A}$ is converted to $\mathrm{B}$ in two reaction steps with a conversion of 50 and $90 \%$, respectively. The pure compound A can be partially replaced by recycling part of the output of the first reactor, which is the only process source in this example. This process source contains impurities of $83.3 \% \mathrm{~mol} / \mathrm{mol}$, product $\mathrm{B}$ being considered as an impurity for recycling, since over a certain 


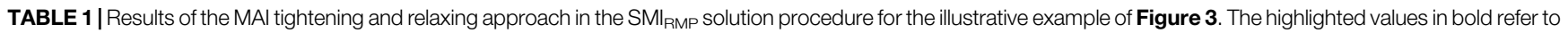
active MAI constraints.

\begin{tabular}{|c|c|c|c|c|c|}
\hline \multicolumn{2}{|c|}{ Tightening constant (\%) } & \multirow{2}{*}{$\begin{array}{l}\text { Impurity level in } \\
\text { the source } \\
(\% \mathrm{~mol})\end{array}$} & \multirow[t]{2}{*}{$\begin{array}{l}\text { Load recycled } \\
(\mathbf{k m o l} / \mathrm{hr})\end{array}$} & \multicolumn{2}{|c|}{$\begin{array}{l}\text { Impurity recycled to the sinks } \\
\text { (kmol/hr) }\end{array}$} \\
\hline & & & & Sink 1 & Sink 2 \\
\hline \multirow[t]{3}{*}{$\mathrm{b}=0$} & After first step & 83.3 & 24 & 0 & 20 \\
\hline & After second step & 83.3 & 0 & 0 & 20 \\
\hline & & & Total: 24 & & \\
\hline \multirow[t]{3}{*}{$b=1$} & After first step & 83.3 & 23.8 & 0 & 19.8 \\
\hline & After second step & 84.1 & 5.8 & 4.9 & 20 \\
\hline & & & Total: 29.6 & & \\
\hline \multirow[t]{3}{*}{$b=2$} & After first step & 83.3 & 23.5 & 0 & 19.6 \\
\hline & After second step & 85 & 12.1 & 10.3 & 20 \\
\hline & & & Total: 35.6 & & \\
\hline \multirow[t]{3}{*}{$b=3$} & After first step & 83.3 & 23.3 & 0 & 19.4 \\
\hline & After second step & 85.8 & 17.5 & 15 & 20 \\
\hline & & & Total: 40.8 & & \\
\hline \multirow[t]{3}{*}{$b=4$} & After first step & 83.3 & 23.0 & 0 & 19.2 \\
\hline & After second step & 85.8 & 17.5 & 15 & 19.8 \\
\hline & & & Total: 40.5 & & \\
\hline \multirow[t]{3}{*}{$b=5$} & After first step & 83.3 & 22.8 & 0 & 19 \\
\hline & After second step & 85.8 & 17.5 & 15 & 19.6 \\
\hline & & & Total: 40.3 & & \\
\hline \multirow[t]{3}{*}{$b=6$} & After first step & 83.3 & 22.5 & 0 & 18.8 \\
\hline & After second step & 85.8 & 17.5 & 15 & 19.4 \\
\hline & & & Total: 40.0 & & \\
\hline
\end{tabular}

amount it can significantly affect the conversion of the two reactions. C represents another type of impurity that is inert to the reaction up to a certain level. The MAIs for replacing part of pure A are 15 and $20 \% \mathrm{~mol} / \mathrm{mol}$ for the two process sinks, respectively.

Applying the $\mathrm{SMI}_{\mathrm{NPM}}$ in this example, the second sink (Sink-2) is ordered first receiving $24 \mathrm{kmol} / \mathrm{h}$ from the process source, and the first sink (Sink-1) is ordered second receiving $18 \mathrm{kmol} / \mathrm{h}$ from the process source. Thus, $\mathrm{OSK}_{\mathrm{NPM}}=\{$ Sink-2, Sink-1 $\}$ and $\mathrm{RT}_{\mathrm{NPM}}=$ $42 \mathrm{kmol} / \mathrm{h}$. Using this information for the $\mathrm{SMI}_{\mathrm{RPM}}$ procedure, the results at different buffer constants are presented in Table $\mathbf{1}$.

When the MAI constraints are not tightened (i.e., $b=0 \%$ ), after the first process sink selection step the maximum recycled amount to Sink- 2 is $24 \mathrm{kmol} / \mathrm{h}$, for which a process constraint becomes active (i.e., in this case the MAI of $\mathrm{SK}_{2}$, highlighted with bold numbers in Table 1). At the second process sink selection step, nothing can be recycled to Sink-1: indeed, even a small recycled amount will cause an increase of the impurity amount in the process source, leading to the violation of the MAI constraint of Sink-2.

By tightening the MAI constraint at the first process sink selection step, typically less amount is recycled. At $b=1 \%$ the reduction is $0.2 \mathrm{kmol} / \mathrm{h}$ at the Sink- 2 selection step. At the Sink-1 selection step, the MAI constraint for Sink-2 is relaxed back to its original value and, thus, it is not anymore active. Since Sink-1 is the last one of the ordered process sinks in this example, no tightening of its MAI constraint is applied here. It is now possible to recycle up to $5.8 \mathrm{kmol} / \mathrm{h}$ to Sink-1, making the MAI constraint of Sink-2 active (i.e., highlighted with bold numbers in Table 1). Therefore, the relatively small reduction in the recycled amount to Sink-2 has resulted in $5.6 \mathrm{kmol} / \mathrm{h}$ more recycled amount. This effect is also observed for other values of the tightening constant. The total recycled amount is maximum at $b=3 \%$. Higher values of tightening the MAI constraint for Sink-2 do not improve the final result anymore because the constraint of the first sink becomes active (i.e., in general because of the limited load and impurity capacity of the process sinks or the limited availability of the process sources). The optimal value of the tightening constant is process specific and requires a sensitivity analysis in every case.

\section{CASE STUDIES AND RESULTS}

In this section, the proposed methodology is demonstrated in two case studies for the design of a direct recycling network with rigorous process models. The first case study is an industrial batch process with two process sources and four process sinks available for integration. It includes typical process units, such as reactors, flash evaporators and decanters. Process information from the non-integrated industrial batch plant for fine chemical production has been used to construct a detailed process model in Aspen Plus ${ }^{\circledR} 8.6$ (www.aspentech.com). This case study is selected to investigate the performance of the proposed method at the complexity level of a typical industrial process in fine chemicals 


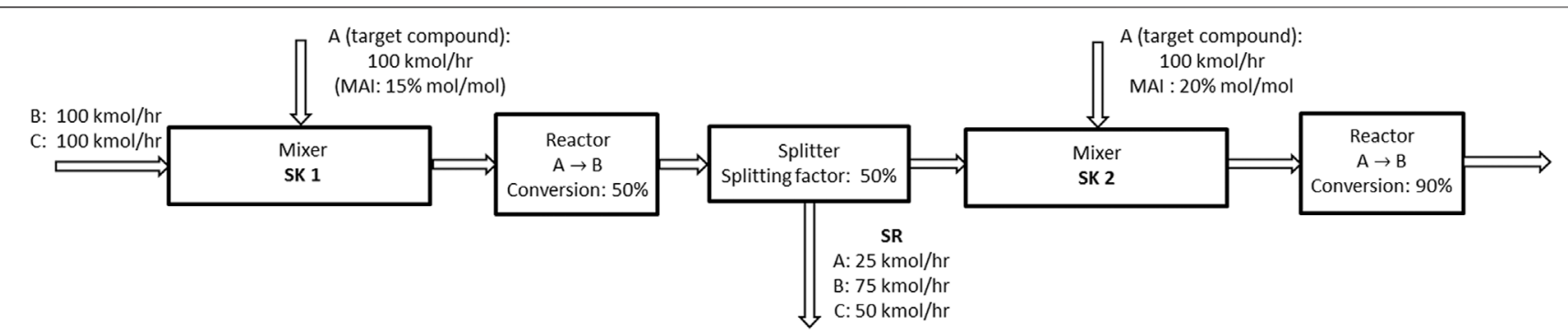

FIGURE 3 | Exemplary process for illustration of tightening and relaxing MAI constraints in the SMlRPM solution procedure. The process sources and sinks are highlighted as well as the two maximum allowable impurity levels. The source has an impurity level of $83.3 \% \mathrm{~mol} / \mathrm{mol}$.

TABLE 2 | Characteristics of process sinks and sources of the case study-1. All loads refer to the target compound (dichloromethane) and the values to $\mathrm{kmol} / \mathrm{batch}$.

\begin{tabular}{lcc} 
Process sinks & Total load capacity & Maximum allowable impurity \\
\hline SK1 & 98.67 & 4.93 \\
SK2 & 75.59 & 3.78 \\
SK3 & 3.12 & 0.47 \\
SK4 & 8.24 & 2.47 \\
\hline Process sources & Load & Impurity amount \\
\hline SR1 & 98.69 & 5.60 \\
SR2 & 142.56 & 52.21 \\
\hline
\end{tabular}

industry, where accurate modelling performance is of high importance because of generally strict product quality specifications. The proposed sequential methodology is discussed from different aspects, including the impact of the tightening constant, the MAI constraints, and the single- and multi-variable based maximization of the recycling amount to the ordered process sinks. The presented cumulative recycling curves provide process insights which can significantly enhance the interpretability of the mass integration solution. Despite the process complexity, it is still possible in this case, although computationally more expensive, to calculate the global optimum of the direct recycling problem. This is then used to assess the performance of the novel sequential methodology regarding its optimality approach.

The second case study is a generic process with three process sources and four process sinks. It includes typical process model units, such as continuously stirred reactors, flash evaporators and decanters and is constructed with the purpose to highlight some particular problematic cases (i.e., with respect to the impurity order of the process sources) that may appear in mass integration under rigorous process modelling conditions and also test the performance of the proposed sequential methodology.

\section{Case Study-1: Demonstrating the Sequential Mass Integration Approach in an Industrial Process \\ Characterization of the Industrial Process}

An industrial batch process to produce a herbicide makes use of a large amount of dichloromethane (target compound) as a solvent and washing agent. The process flowsheet is illustrated in Figure 4. The reactor models comprise detailed kinetic models on the basis of calorimetric and spectroscopic absorption data available from laboratory experiments (Chatterjee, 2012). Similarly, the decanter and crystallizer thermodynamic models represent the industrial process performance.

The fresh target compound is required by the two reactors and two mixers (i.e., mixers two and three in Figure 4), which are the four process sinks. Two waste streams of the non-integrated process are available for direct recycling. These are the two process sources in this case study. The characteristics of all process sinks and sources are presented in Table 2. A detailed description of the process conditions and modelling assumptions can be found in the Electronic Supporting Information, together with a complete stream table of the process (Supplementary Table S1).

On the basis of the characteristics of the process sources and sinks, it is possible to solve the preemptive-goal programming problem according to the procedure presented in Figure 1 to obtain the $\mathrm{OSK}_{\mathrm{NPM}}, \mathrm{RN}_{\mathrm{NPM}}$ and the corresponding cumulative curve up to the $\mathrm{RT}_{\mathrm{NPM}}$. Using these results, the $\mathrm{SMI}_{\mathrm{RPM}}$ can be applied to realize a recycle network with rigorous process modelling.

\section{Results and Discussion of the SMI $\mathrm{RPM}_{\mathrm{R}}$ Application}

Both versions of the $\mathrm{SMI}_{\mathrm{RPM}}$ approach have been applied for each process sink selection step, namely the single variable sequential and the multi-variable simultaneous maximization of the respective recycling amount. The results are reported in Figure 5 in the form of cumulative recycling curves and in Table 3 including the respective source-to-sink connections at each step. The total amount recycled in the various $\mathrm{SMI}_{\mathrm{RPM}}$ versions is lower than the $\mathrm{RT}_{\mathrm{NPM}}$. This is expected, as in this case the global optimum by solving the respective NLP problem (i.e., considering simultaneously the superstructure of all eight source-to-sink connections, solved through the SQP algorithm (Biegler et al., 1997) performed by Aspen Plus ${ }^{\circledR}$ 8.6) is also lower than the $\mathrm{RT}_{\mathrm{NPM}}$. As can be seen in Table 3, the multi-variable simultaneous approach at each process selection step has a very small optimality gap with respect to the NLP global optimum, but also the single-variable sequential approach approximation is satisfactory considering also the significantly lower computational effort. Additionally, both approaches require 


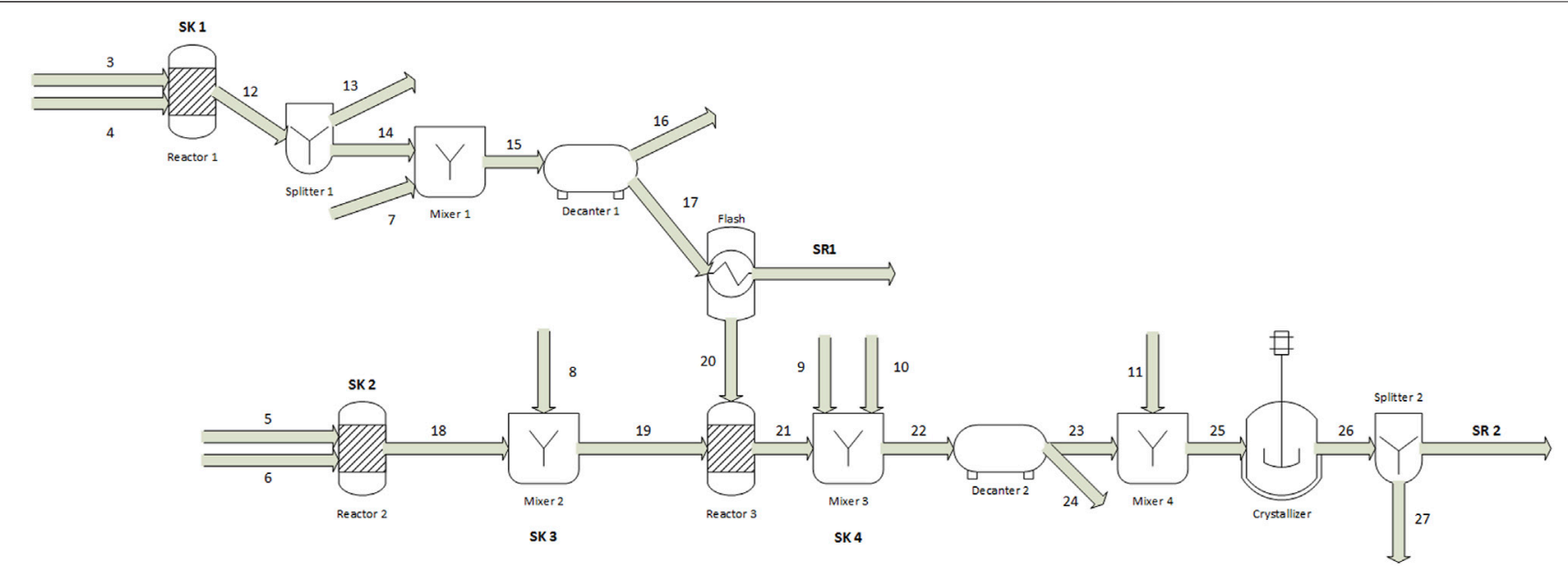

FIGURE 4 | Process flowsheet for the case study-1 highlighting all process sinks and sources.

TABLE 3 $\mid S_{\text {NPM }}, \mathrm{SMl}_{\mathrm{RPM}}$ and global optimum results for the case study-1. For each sink the respective sources recycling to that sink are shown. The results for the singlevariable sequential and multi-variable simultaneous recycling maximisation approaches at each process sink selection step are reported at their optimal tightening constant values. The reported values refer to $\mathrm{kmol} / \mathrm{batch}$. MAl of the first sink is $5 \% \mathrm{~mol} / \mathrm{mol}$.

\begin{tabular}{|c|c|c|c|c|}
\hline \multirow[t]{2}{*}{ OSK $_{\text {NPM }}$} & \multirow[t]{2}{*}{$\mathrm{SMI}_{\mathrm{NPM}}$} & \multirow{2}{*}{ 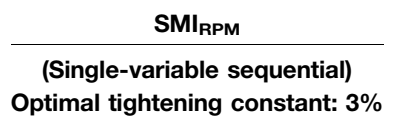 } & \multirow{2}{*}{ 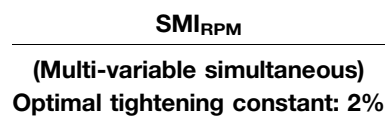 } & \multirow[t]{2}{*}{ Global optimum } \\
\hline & & & & \\
\hline SK1 & SR1: 86.99 & SR1: 70.48 & SR1: 71.04 & SR1: 71.32; SR2: 0.14 \\
\hline SK2 & SR1: 11.69; SR2: 8.51 & SR1: 11.69; SR2: 7.64 & SR1: 24.01; SR2: 5.52 & SR1: 22.26; SR2: 5.83 \\
\hline SK4 & SR2: 6.75 & SR2: 6.21 & SR2: 6.29 & SR1: 1.40; SR2: 6.13 \\
\hline SK3 & SR2: 1.28 & SR2: 1.21 & SR2: 1.21 & SR2: 1.21 \\
\hline Total & 115.22 & 97.23 & 108.07 & 108.29 \\
\hline
\end{tabular}

the solution of optimization problems with one or few variables; therefore, they need less computational effort than the optimization of other multi-variable superstructures presented in literature (Ahmetovic et al., 2015). It should be noted that the optimal values for the two versions of the $\mathrm{SMI}_{\mathrm{RPM}}$ are obtained for different values of the tightening constant. It should also be noted that the NLP global optimum has a different sink-to-source connectivity pattern compared to the $\mathrm{SMI}_{\mathrm{RPM}}$ solution (i.e., the split of SR2 between SK1 and SK3 leads to a more comple process design with only a small benefit in terms of the total recycled amount).

The interaction of the rigorous process modelling with the direct recycling can be easily interpreted following the proposed methodology. For instance, in the case of the sequential approach with a tightening constant of $0 \%$, the recycling of the impurity from the first source to the first sink has also an influence on the thermodynamic equilibria of the first decanter and the flash evaporator; these two units are mostly responsible for the increase of the impurity degree in the first source, from 5.67 to $6.83 \% \mathrm{~mol} / \mathrm{mol}$, and, consequently, for the difference with the recycled load obtained with the $\mathrm{SMI}_{\mathrm{NPM}}$. At the second step, the thermodynamic equilibrium in the second decanter is mostly responsible for the increase of the impurity level of the second source, which is finally $37.61 \%$ from the initial $36.62 \% \mathrm{~mol} / \mathrm{mol}$.

In the multi-variable simultaneous optimization approach with a tightening constant of $0 \%$, the availability of the purest source allows a higher recycled load to the second sink compared to the one recycled with the sequential approach $(28.74$ vs $19.61 \mathrm{kmol})$, resulting in a higher $\mathrm{RT}_{\mathrm{RPM}}(100.89 \mathrm{kmol}$ compared to $91.76 \mathrm{kmol}$, respectively).

The cumulative curves of Figure 5 clearly show the positive effect of tightening and relaxing the MAI constraints of the process sinks. Considering the two optimal tightening constants, a slight decrease in the recycled loads at the first process sink selection step allows to increase the final recycled amount; this effect can be clearly identified already from the third process sink selection step up to the final $\mathrm{RT}_{\mathrm{RPM}}$ value in both the single-variable sequential and multi-variable simultaneous optimization approaches.

The multi-variable simultaneous optimization approach obtained consistently better results than the single-variable sequential approach for all values of the tightening constant, as can be seen in Figure 6. As previously explained, the positive effect of the tightening constant is visible only until a certain level (i.e., approximately around $b=3 \%$ for both approaches in this case). 


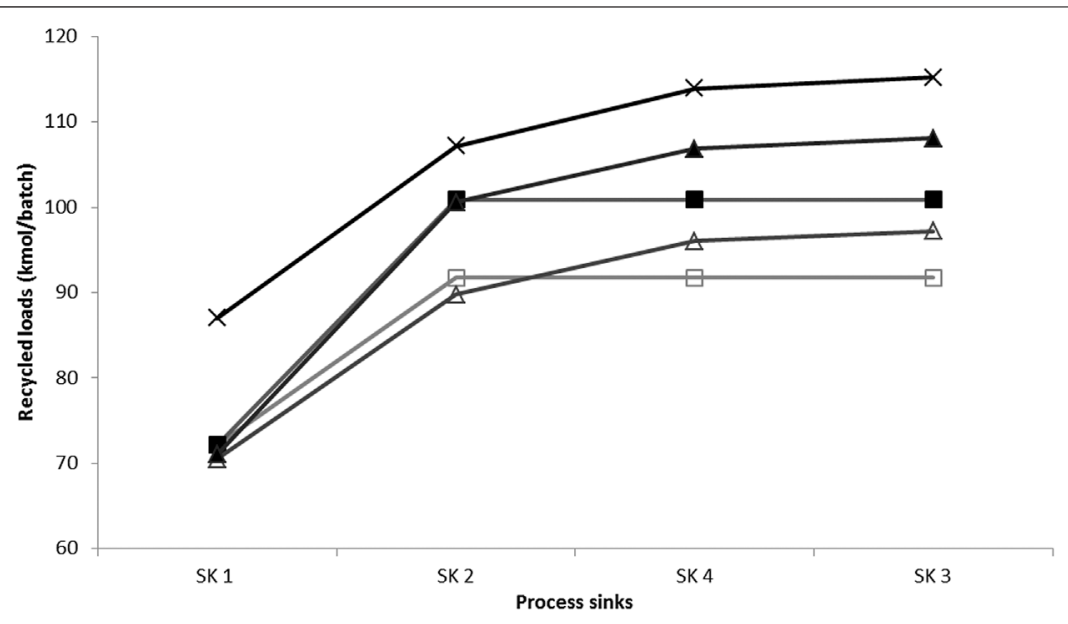

FIGURE 5 | Cumulative curve of the optimal recycled loads in the case study- 1 (SMINPM: crosses; $\mathrm{SMl}_{\mathrm{RPM}}$ at $\mathrm{b}=0 \%$ for single-variable sequential optimization at each process selection step: hollow squares; $\mathrm{SMI}_{\mathrm{RPM}}$ at $\mathrm{b}=0 \%$ for multi-variable simultaneous optimization at each process selection step: full squares; SMI RPM at optimal $b=3 \%$ for single-variable sequential optimization at each process selection step: hollow triangles; $S M l_{R P M}$ at optimal $b=2 \%$ for multi-variable simultaneous optimization at each process selection step: full triangles).

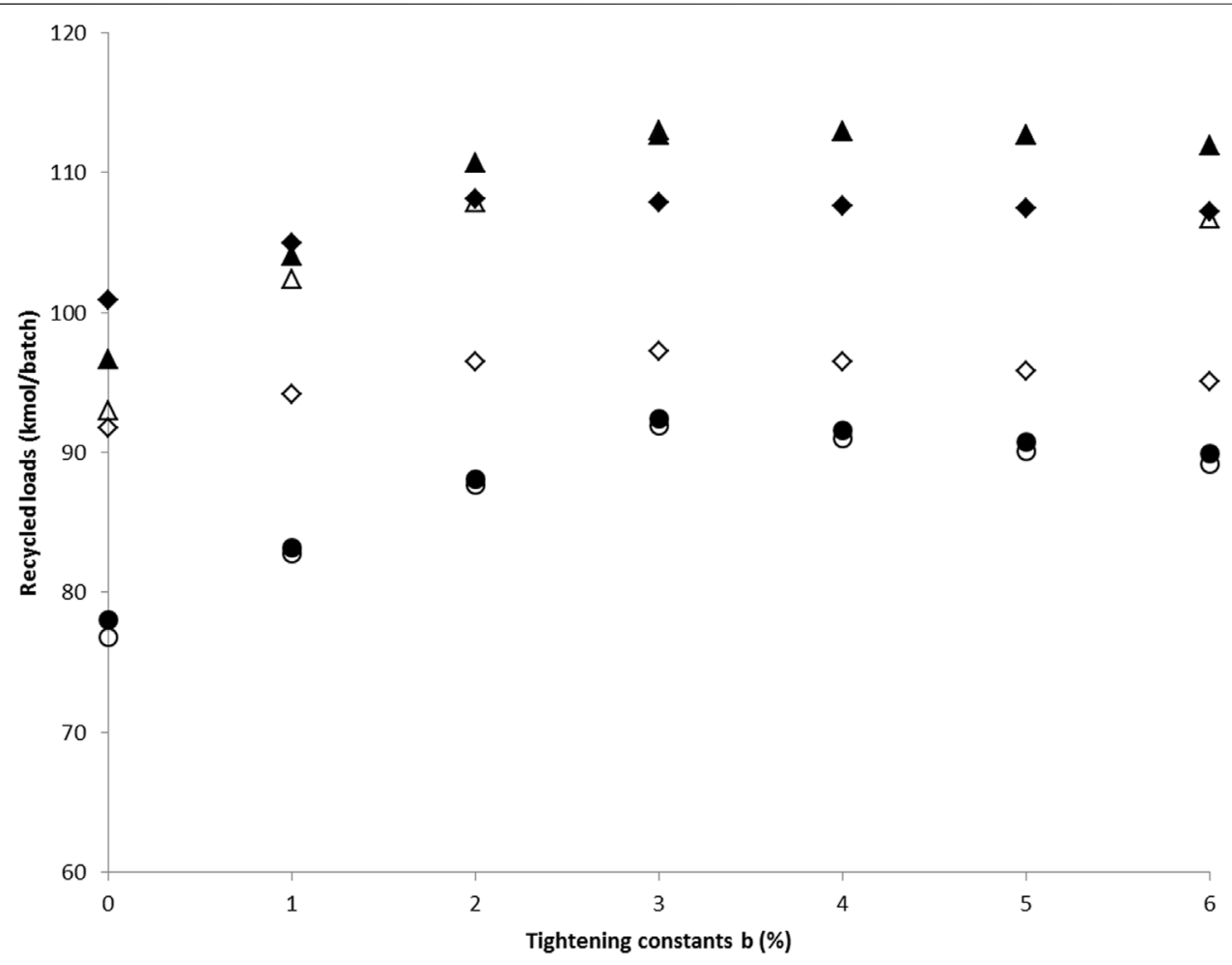

FIGURE 6 | Total recycled loads of the SMI RPM method in the case study-1 for different tightening constants and MAI constraints in SK1 (single-variable sequential optimization at each process selection step and MAI constraint 1\%: hollow circles; single-variable sequential optimization at each process selection step and MAI constraint 5\%: hollow rhombuses; single-variable sequential optimization at each process selection step and MAl constraint 10\%: hollow triangles; multi-variable simultaneous optimization at each process selection step and MAI constraint 1\%: full circles; multi-variable simultaneous optimization at each process selection step and MAI constraint 5\%: full rhombuses; multi-variable simultaneous optimization at each process selection step and MAl constraint 10\%: full triangles).

The same analysis of the $\mathrm{SMI}_{\mathrm{RPM}}$ approach is performed with an additional process constraint, other than the MAI constraints of the process sinks (i.e., a design specification over the final quantity of the product). Similar trends have been observed and are discussed in more detail in the Electronic Supporting Information (Supplementary Figure S1), together with an 
TABLE 4 | Characteristics of process sinks and sources of the case study-2.

\begin{tabular}{lcc} 
Process sinks & $\begin{array}{c}\text { Total load capacity } \\
\text { (kmol/hr) }\end{array}$ & $\begin{array}{c}\text { Maximum allowable impurity } \\
\text { (kmol/hr) }\end{array}$ \\
\hline SK1 & 20 \\
SK2 & 15 \\
SK3 & 15 \\
SK4 & 15 \\
\hline Process sources & Load (kmol/hr) \\
SR1 & 10 \\
SR2 & 20 \\
SR3 & 3.0 \\
& 30
\end{tabular}

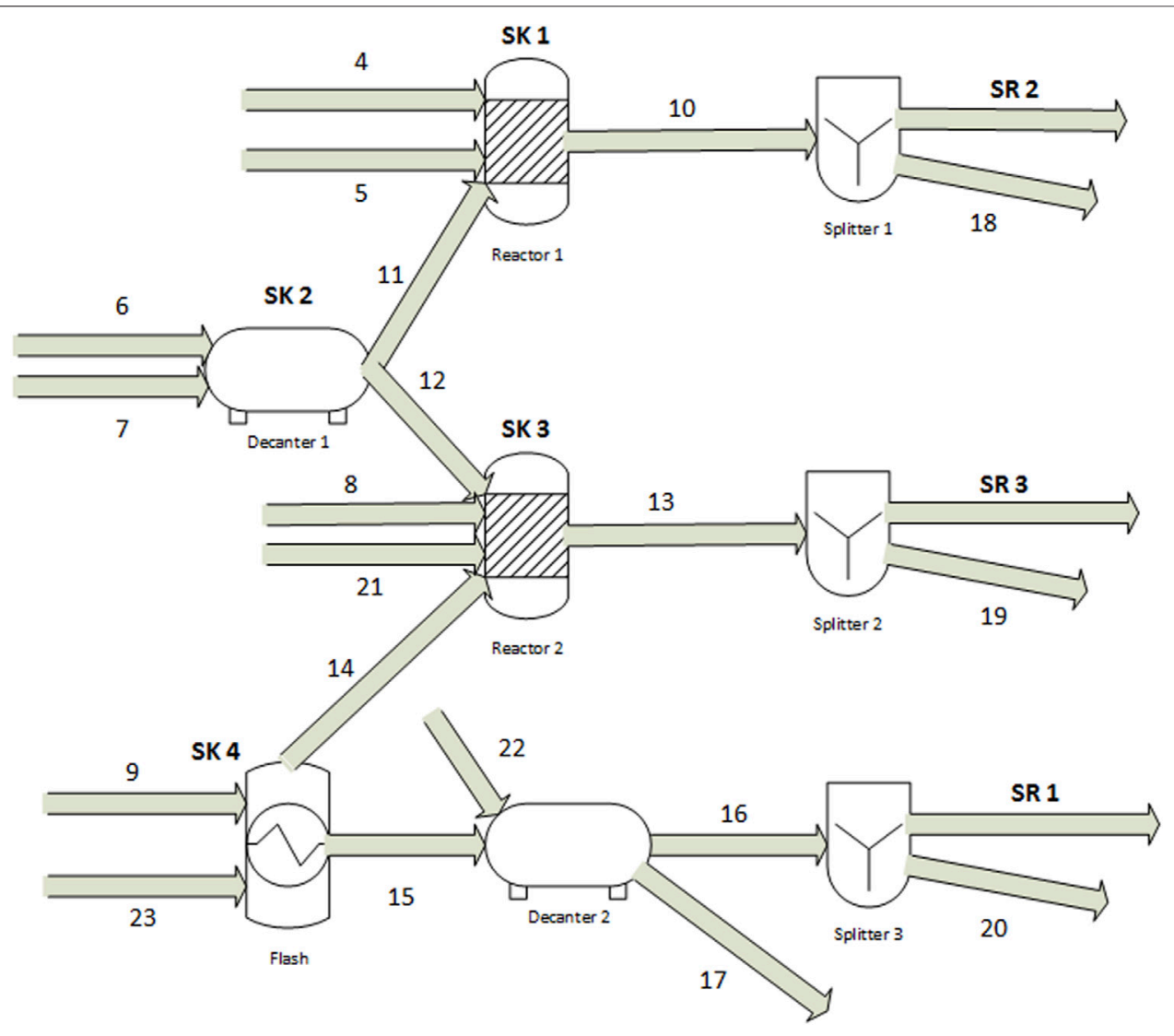

FIGURE 7 | Process flowsheet for the case study-2 highlighting all process sinks and sources.

analysis of the impact of different MAI constraints in the $\mathrm{SMI}_{\mathrm{RPM}}$ (Supplementary Figures S2, 3 and Table S2).

\section{Case Study-2: Highlighting a Special Case for the Sequential Mass Integration Approach in a Generic Case Study}

The process flowsheet of the generic case study is illustrated in Figure 7, highlighting the three process sources and four process sinks. For this process, a direct recycling network is to be designed following the $\mathrm{SMI}_{\mathrm{RPM}}$ approach. It is desired to minimize the use of the fresh amount of the target compound $\mathrm{A}$ in the streams 4,6 , eight and nine through the recycling of the process sources 1, two and 3. Typical compounds of the Aspen Plus database were included in this case study to safeguard the reliability of the thermodynamic and process models. The characteristics of all the process units ( 2 reactors, 2 decanters and one flash evaporator) and the stream table from the Aspen Plus 8.6 simulation are reported in the Electronic Supporting Information (Supplementary Table S3). In Table 4, the characteristics of the process sink and sources are summarized.

On the basis of this information, it is possible to solve the preemptive-goal programming problem according to the 
TABLE 5|SMI NPM $_{\text {, SMI }}$ RPM and global optimum results for the case study-2. For each sink the respective sources recycling to that sink are shown. The results for the singlevariable sequential and multi-variable simultaneous recycling maximisation approaches at each process sink selection step are reported at their optimal tightening constant values. All reported values refer to $\mathrm{kmol} / \mathrm{hr}$.

\begin{tabular}{|c|c|c|c|c|}
\hline \multirow[t]{2}{*}{ OSK $_{\text {NPM }}$} & \multirow[t]{2}{*}{$\mathrm{SMI}_{\mathrm{NPM}}$} & \multirow{2}{*}{$\frac{\mathrm{SMI}_{\mathrm{RPM}}}{\text { (Sequential) Tightening: } 5 \%}$} & $\mathrm{SMI}_{\mathrm{RPM}}$ & \multirow[t]{2}{*}{ Global optimum } \\
\hline & & & (Simultaneous) Tightening: $5 \%$ & \\
\hline SK4 & SR1: 4.83 SR2: 10.16 & SR1: 0.5 SR2: 10.16 & SR2: 10.86 & SR3: 8.39 \\
\hline SK2 & SR1: 5.16 SR2: 5.5 & SR1: 0.63 SR2: 5.5 & SR2: 6.37 & SR1: 0.74 SR2: 6.33 \\
\hline SK3 & SR2: 4.33 SR3: 0.18 & SR2: 4.25 & SR2: 2.77 SR3: 1.11 & SR1: 6.73 SR2: 2.61 \\
\hline SK1 & SR3: 2.27 & SR3: 2.25 & SR3: 2.25 & SR1: 2.53 SR2: 0.96 SR3: 0.97 \\
\hline Total & 32.43 & 23.29 & 23.36 & 29.26 \\
\hline
\end{tabular}

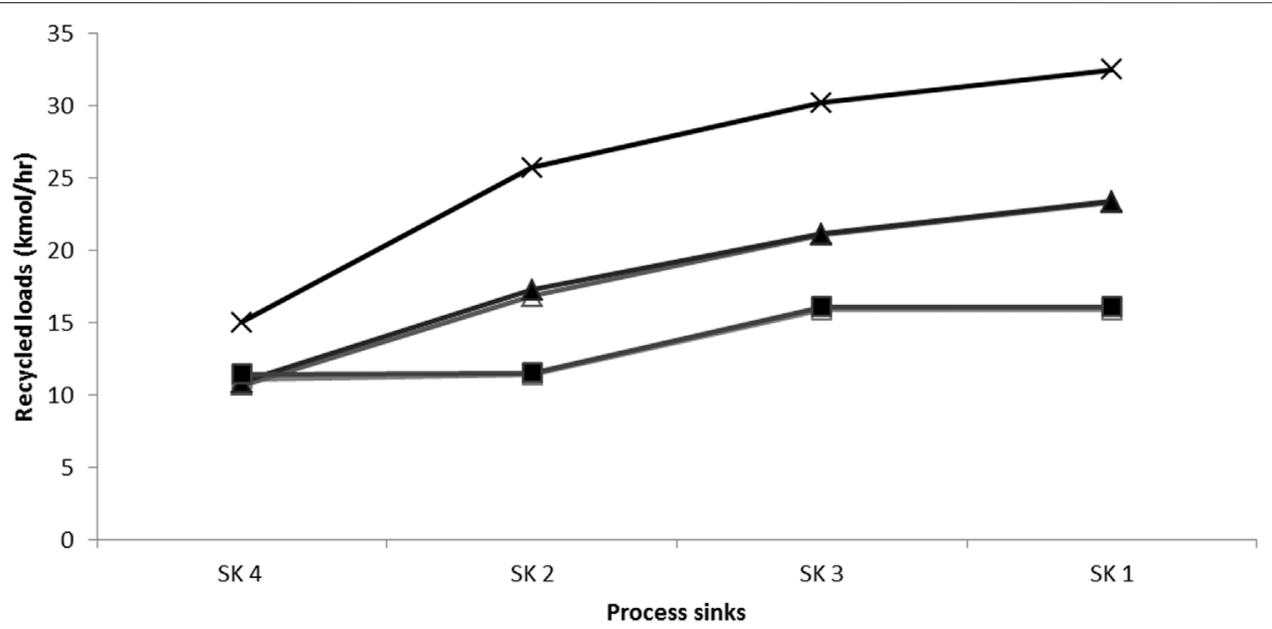

FIGURE 8 | Cumulative curve of the optimal recycled loads in the case study-2 $\left(\mathrm{SMI}_{\mathrm{NPM}}\right.$ : crosses; $\mathrm{SMI} \mathrm{RPM}_{\mathrm{R}}$ at $\mathrm{b}=0 \%$ for single-variable sequential optimization at each process selection step: hollow squares; $S M_{\mathrm{RPM}}$ at $\mathrm{b}=0 \%$ for multi-variable simultaneous optimization at each process selection step: full squares; $\mathrm{SM} \mathrm{R}_{\mathrm{RPM}}$ at optimal $b=5 \%$ for single-variable sequential optimization at each process selection step: hollow triangles; $\mathrm{SM} \mathrm{RPM}_{\mathrm{RP}}$ at optimal $\mathrm{b}=5 \%$ for multi-variable simultaneous optimization at each process selection step: full triangles).

procedure presented in Figure 1 to obtain the $\mathrm{OSK}_{\mathrm{NPM}}, \mathrm{RN}_{\mathrm{NPM}}$ and the corresponding cumulative curve up to the $\mathrm{RT}_{\mathrm{NPM}}$. Using these results, the $\mathrm{SMI}_{\mathrm{RPM}}$ can be applied to realize a recycle network under rigorous process modelling.

The resulting cumulative curves are presented in Figure 8 for two levels of tightening the MAI constraints, namely $b=0 \%$ and $b=5 \%$, which is the optimum value among those tested in this case study (Supplementary Figure S4 in Electronic Supporting Information).

As already explained, tightening the MAI constraints decreases the recycled amount at the specific process sink selection step but it can improve the total amount recycled in the next sinks. This second effect prevails until a maximum point, located approximately at $b=5 \%$ for both the single-variable sequential and the multi-variable simultaneous versions of the $\mathrm{SMI}_{\mathrm{RPM}}$ approach. Moreover, in this case study, these two approaches present very similar results overall.

A deeper analysis of the process shows that in all the recycling scenarios imposed by the $\mathrm{SMI}_{\mathrm{RPM}}$, no phase splitting occurs in the second decanter (i.e., phase splitting takes place in the base case non-integrated scenario). Consequently, the purest source SR1 coming out of the second decanter becomes the most impure source already after the first step (i.e., after recycling the sources SR1 and SR2 to the sink SK4). For instance, in the sequential approach with tightening constant $\mathrm{b}=0 \%$, the impurity level of the first source changes from 17.2 to $92 \% \mathrm{~mol} / \mathrm{mol}$. As a consequence, in the single-variable sequential optimization approach of the $\mathrm{SMI}_{\mathrm{RPM}}$, the first impure source to be optimized is SR1. In this case, the inversion of the impurity order of the process sources is the main reason for the difference between the $\mathrm{SMI}_{\mathrm{NPM}}$ and $\mathrm{SMI}_{\mathrm{RPM}}$ results in this case study (Table 5). Again, the integration designs obtained by the sequential approaches are less complex compared to the NLP global optimum solution. However, in this case the preference for these simpler designs is not straightforward because of the optimality gap (approximately $20 \%)$. Thus, additional metrics with respect to operability, safety and flexibility of the designs obtained by the sequential method would be needed to justify a decision in their favor compared to NLP global optimum solutions, if these can be obtained under rigorous process simulation conditions.

Comparing in Table 5 the $\mathrm{SMI}_{\mathrm{RPM}}$ solutions with the global optimum (i.e., obtained by the SQP method in Aspen Plus ${ }^{\circledR} 8.6$ ), a higher optimality gap is observed than in the first case study. The 


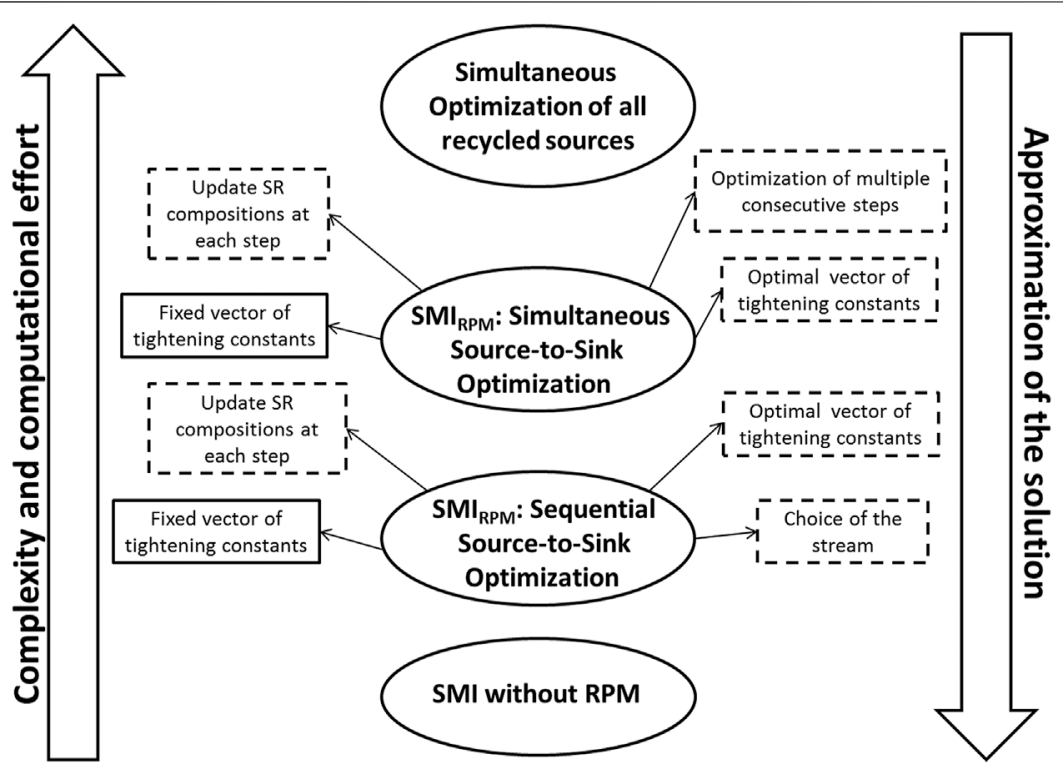

FIGURE 9 | Summary of the algorithms used in this work to realize direct recycling networks with and without rigorous process modelling. Possibilities of further improvement of the approaches presented herein are provided in boxes with dashed contour. The applied methodologies and the potential further improvements are compared according to their level of approximation of the global optimum and the required computational effort.

sinks-to-sources connectivity of the global optimum is such that two liquid phases are still separated by the second decanter, and thus the original order of impurity of the process sources is maintained. This is the reason that the $\mathrm{RT}_{\mathrm{NPM}}$ solution is closer to the global optimum solution in this case study. This is a very particular feature of this case study that can lead however to a more general statement: if the impurity order of the process sources is maintained when the direct recycling network is realized under rigorous process simulation conditions, then a better approximation of the global optimum can be reached by the $\mathrm{SMI}_{\mathrm{RPM}}$ method.

\section{CONCLUSION}

A sequential approach has been proposed to realize a mass integration network through direct recycling using rigorous process modelling. The methodology requires as initial values, the sink-to-source connectivity characterizing the optimal ordering of the process sinks. This is obtained by solving a linear preemptive-goal programming problem, which can be decomposed in a succession of linear optimization problems. In the linear optimization algorithm, the composition of the process sources is assumed not to change after closing the recycling loops.

The source-to-sink connectivity is then used under rigorous process modelling conditions, representing in the general case nonlinear input-output relations for all process units. When recycling loops are realized, process constraint violations may occur, which are then fixed by a single-variable sequential or multi-variable simultaneous optimization approach at each process sink selection step. An important aspect for both approaches is to obtain an optimal degree of integration at earlier process selection steps. In the present work, this is achieved by a tightening and relaxing procedure for the MAI constraints of the process sinks leading to satisfactory approximations of the global optimum with significantly less computational effort. Optimality gaps from 0.2 to $10 \%$ have been observed, if the impurity order of the process sources is maintained when the direct recycling network is realized under rigorous process simulation conditions. Bigger optimality gaps (approximately 20\%) are observed if this condition does not hold. Thus, the proposed method should be applied to more case studies of various complexity to infer a distribution of the optimality gap and quantify the computational benefit.

In addition to the satisfactory optimality gap, a novelty of this sequential mass integration method for direct recycling problems compared to state-of-the-art approaches is that it can be easily integrated in advanced process simulators (e.g., Aspen Plus) in the sense that it neither requires process model simplifications nor the transfer of the process model into mathematical modelling and optimisation software. This advantage comes with a trade-off between reduced computational effort and the optimality gap (e.g., compared to NLP superstructure solutions). Moreover, the results of the method can be more easily interpreted by the decision makers (e.g., process engineers), who can follow and possibly interact with the method results at every step of the integration problem, rather than only examining the final solution.

It should be noted that considering the general superstructure approach for synthesis of mass integration networks (e.g., water networks) in the presence of regeneration units, the presented algorithm can be directly applied in the case of one contaminant 
and fixed composition from the regeneration units. This would, however, denote a rather simple application of the approach, since, in this case, the process sources would be the outlet streams of the regeneration units, with constant composition independent from the background process effects. If, however, instead of fixed compositions, only fixed contaminant removal ratios are assumed for the regeneration units and minimum fresh source consumption as objective function, the sequential approaches presented here can be used to take account of the changing source compositions due to the process units and the regeneration units. Even if this case is less complex than directly considering a rigorous process model, and the computational benefit compared to the standard superstructure approaches may not be as big, it is mentioned here to designate the compatibility of the approach with some standard problem formulations in this field.

The different versions of the sequential integration methodology presented herein are summarized in Figure 9, providing also some directions for further improvement. For instance, the order of process sinks can be dynamically updated at each process sink selection step considering the new process source compositions after realizing the recycling network for that specific process sink. Additionally, an optimal set of tightening constants can be derived for the set of process sinks. Furthermore, the simultaneous optimization methodology can be applied to more than one process sinks, for instance by grouping consecutive sinks of the optimal order obtained with $\mathrm{SMI}_{\mathrm{NPM}}$. It is expected that such methodology variations can further reduce the optimality gap to the global optimum derived by

\section{REFERENCES}

Adekola, O., and Majozi, T. (2011). Wastewater Minimization in Multipurpose Batch Plants with a Regeneration Unit: Multiple Contaminants. Comput. Chem. Eng. 35, 2824-2836. doi:10.1016/ j.compchemeng.2011.04.008

Ahmetovic, E., and Grossmann, I. E. (2011). Global Superstructure Optimization for the Design of Integrated Process Water Networks. AIChE J. 57, 434-457. doi:10.1002/aic.12276

Ahmetovic, E., Ibric, N., Kravanja, Z., and Grossmann, I. E. (2015). Water and Energy Integration: A Comprehensive Literature Review of Non-isothermal Water Network Synthesis. Comput. Chem. Eng. 82, 144-171. doi:10.1016/ j.compchemeng.2015.06.011

Ahmetovic, E., Ibric, N., and Kravanja, Z. (2014). Optimal Design for HeatIntegrated Water-Using and Wastewater Treatment Networks. Appl. Energ. 135, 791-808. doi:10.1016/j.apenergy.2014.04.063

Ahmetovic, E., and Kravanja, Z. (2013). Simultaneous Synthesis of Process Water and Heat Exchanger Networks. Energy 57, 236-250. doi:10.1016/ j.energy.2013.02.061

Alhajri, I., Elkamel, A., Albahri, T., and Douglas, P. L. (2008). A Nonlinear Programming Model for Refinery Planning and Optimisation with Rigorous Process Models and Product Quality Specifications. Ijogct 1, 283-307. doi:10.1504/ijogct.2008.019846

Argaez, A. A., Kokossis, A. C., and Smith, R. (2007). A Conceptual Decomposition of MINLP Models for the Design of Water-Using Systems. Ijep 29, 177-205. doi:10.1504/ijep.2007.012803

Baykasoglu, A. (2005). Preemptive Goal Programming Using Simulated Annealing. Eng. Optimization 37, 49-63. doi:10.1080/0305215042000268606

Biegler, L. T., Grossmann, I. E., and Westerberg, A. W. (1997). Systematic Methods for Chemical Process Design. considering the whole recycling superstructure, but nevertheless at a gradually higher computational effort.

\section{DATA AVAILABILITY STATEMENT}

The original contributions presented in the study are included in the article and the Supplementary Material, further inquiries can be directed to the corresponding author.

\section{AUTHOR CONTRIBUTIONS}

FM conducted the research as part of hid PhD studies at ETH Zurich under the supervision of SP and KH. FM wrote the manuscript together with SP.

\section{ACKNOWLEDGMENTS}

The authors acknowledge TU Wien Bibliothek for financial support through its Open Access Funding Program.

\section{SUPPLEMENTARY MATERIAL}

The Supplementary Material for this article can be found online at: https://www.frontiersin.org/articles/10.3389/fceng.2021.785179/ full\#supplementary-material

Boix, M., Montastruc, L., Pibouleau, L., Azzaro-Pantel, C., and Domenech, S. (2011). A Multiobjective Optimization Framework for Multicontaminant Industrial Water Network Design. J. Environ. Manage. 92, 1802-1808. doi:10.1016/j.jenvman.2011.02.016

Caballero, J. A., and Grossmann, I. E. (2008). "Rigorous Flowsheet Optimization Using Process Simulators and Surrogate Models," in 18th European Symposium on Computer Aided Process Engineering. Editors B. Braunschweig and X. Joulia, 25, 551-556. doi:10.1016/s1570-7946(08) 80097-1

Chatterjee, S. (2012). Modelling of Multi-step Batch Production of Specialty Chemicals Using Experimental and Industrial Data. ETH Zurich: Master thesis.

El-Halwagi, M. M., Gabriel, F., and Harell, D. (2003). Rigorous Graphical Targeting for Resource Conservation via Material Recycle/reuse Networks. Ind. Eng. Chem. Res. 42, 4319-4328. doi:10.1021/ie030318a

El-Halwagi, M. M., and Manousiouthakis, V. (1990). Automatic Synthesis of MassExchange Networks with Single-Component Targets. Chem. Eng. Sci. 45, 2813-2831. doi:10.1016/0009-2509(90)80175-e

El-Halwagi, M. M., and Manousiouthakis, V. (1989). Synthesis of Mass Exchange Networks. Aiche J. 35, 1233-1244. doi:10.1002/aic.690350802

El-Halwagi, M. M. (2006). Process Integration. San Diego, CA 92101-4495 USA: Elsevier Academic Press Inc.

Faria, D. C., and Bagajewicz, M. J. (2008). A New Approach for the Design of Multicomponent Water Wastewater Networks. 18th European Symposium on Computer Aided Process Engineering - ESCAPE 18 - Bertrand Braunschweig and Xavier Joulia (Editors).

Foo, D. C. Y., Manan, Z. A., and Tan, Y. L. (2006). Use cascade Analysis to Optimize Water Networks. Chem. Eng. Prog. 102, 45-52.

Gabriel, F. B., and El-Halwagi, M. M. (2005). Simultaneous Synthesis of Waste Interception and Material Reuse Networks: Problem Reformulation for Global Optimization. Environ. Prog. 24, 171-180. doi:10.1002/ep.10081 
Gonws, J. F., Majozi, T., Foo, D. C. Y., Chen, C. L., and Lee, J. Y. (2010). Water Minimization Techniques for Batch Processes. Ind. Eng. Chem. Res. 49, 8877-8893. doi:10.1021/ie100130a

Halim, I., and Srinivasan, R. (2011). Sequential Methodology for Integrated Optimization of Energy and Water Use during Batch Process Scheduling. Comput. Chem. Eng. 35, 1575-1597. doi:10.1016/j.compchemeng.2011.05.009

Halim, I., and Srinivasan, R. (2009). Sequential Methodology for Scheduling of HeatIntegrated Batch Plants. Ind. Eng. Chem. Res. 48, 8551-8565. doi:10.1021/ie900367j

Hallale, N. (2002). A New Graphical Targeting Method for Water Minimisation. Adv. Environ. Res. 6, 377-390. doi:10.1016/s1093-0191(01)00116-2

Handani, Z. B., Quaglia, A., and Gani, R. (2015). "Synthesis and Design of Integrated Process and Water Networks," in 12th International Symposium on Process Systems Engineering. Editors K. V. Gernaey, J. K. Huusom, and R. Gani (Amsterdam: Elsevier Science Bv), Vol. 37, 875-880. doi:10.1016/b9780-444-63578-5.50141-9

Ibric, N., Ahmetovic, E., and Kravanja, Z. (2014). Simultaneous Optimization of Water and Energy within Integrated Water Networks. Appl. Therm. Eng. 70, 1097-1122.

Jiménez-Gutiérrez, A., Lona-Ramírez, J., Ponce-Ortega, J. M., and El-Halwagi, M. (2014). An MINLP Model for the Simultaneous Integration of Energy, Mass and Properties in Water Networks. Comput. Chem. Eng. 71, 52-66. doi:10.1016/ j.compchemeng.2014.07.008

Karuppiah, R., and Grossmann, I. E. (2006). Global Optimization for the Synthesis of Integrated Water Systems in Chemical Processes. Comput. Chem. Eng. 30, 650-673. doi:10.1016/j.compchemeng.2005.11.005

Kheireddine, H., Dadmohammadi, Y., Deng, C., Feng, X., and El-Halwagi, M. (2011). Optimization of Direct Recycle Networks with the Simultaneous Consideration of Property, Mass, and Thermal Effects. Ind. Eng. Chem. Res. 50, 3754-3762. doi:10.1021/ie1012272

Majozi, T. (2005). An Effective Technique for Wastewater Minimisation in Batch Processes. J. Clean. Prod. 13, 1374-1380. doi:10.1016/j.jclepro.2005.04.016

Majozi, T., Brouckaert, C. J., and Buckley, C. A. (2006). A Graphical Technique for Wastewater Minimisation in Batch Processes. J. Environ. Manage. 78, 317-329. doi:10.1016/j.jenvman.2005.04.026

Malik, S. N., Bahri, P. A., and Vu, L. T. T. (2015). "Superstructure Development, Simulation and Optimization of Desalination Systems Using Aspen Custom Modeler," in 12th International Symposium on Process Systems Engineering. Editors K. V. Gernaey, J. K. Huusom, and R. Gani, 37, 383-388. doi:10.1016/ b978-0-444-63578-5.50059-1

Manan, Z. A., Tan, Y. L., and Foo, D. C. Y. (2004). Targeting the Minimum Water Flow Rate Using Water cascade Analysis Technique. Aiche J. 50, 3169-3183. doi:10.1002/aic.10235
Marchione, F., Papadokonstantakis, S., and Hungerbuehler, K. (2016). Sequential Ordering Algorithm for Mass Integration: The Case of Direct Recycling. Aces 06, 158-182. doi:10.4236/aces.2016.62018

Nápoles-Rivera, F., Ponce-Ortega, J. M., El-Halwagi, M. M., and JiménezGutiérrez, A. (2012). Global Optimization of Wastewater Integration Networks for Processes with Multiple Contaminants. Environ. Prog. Sustain. Energ. 31, 449-458. doi:10.1002/ep.10557

Porzio, G. F., Colla, V., Fornai, B., Vannucci, M., Larsson, M., and Stripple, H. (2016). Process Integration Analysis and Some Economic-Environmental Implications for an Innovative Environmentally Friendly Recovery and Pretreatment of Steel Scrap. Appl. Energ. 161, 656-672. doi:10.1016/ j.apenergy.2015.08.086

Seid, E. R., and Majozi, T. (2014). Optimization of Energy and Water Use in Multipurpose Batch Plants Using an Improved Mathematical Formulation. Chem. Eng. Sci. 111, 335-349. doi:10.1016/j.ces.2014.02.036

Sorin, M., and Bédard, S. (1999). The Global Pinch point in Water Reuse Networks. Process Saf. Environ. Prot. 77, 305-308. doi:10.1205/ 095758299530189

Tudor, R., and Lavric, V. (2011). Dual-objective Optimization of Integrated Water/ wastewater Networks. Comput. Chem. Eng. 35, 2853-2866. doi:10.1016/ j.compchemeng.2011.04.010

Wang, Y. P., and Smith, R. (1994). Wastewater Minimisation. Chem. Eng. Sci. 49, 981-1006. doi:10.1016/0009-2509(94)80006-5

Conflict of Interest: The authors declare that the research was conducted in the absence of any commercial or financial relationships that could be construed as a potential conflict of interest.

Publisher's Note: All claims expressed in this article are solely those of the authors and do not necessarily represent those of their affiliated organizations, or those of the publisher, the editors and the reviewers. Any product that may be evaluated in this article, or claim that may be made by its manufacturer, is not guaranteed or endorsed by the publisher.

Copyright (C) 2021 Marchione, Hungerbuehler and Papadokonstantakis. This is an open-access article distributed under the terms of the Creative Commons Attribution License (CC BY). The use, distribution or reproduction in other forums is permitted, provided the original author(s) and the copyright owner(s) are credited and that the original publication in this journal is cited, in accordance with accepted academic practice. No use, distribution or reproduction is permitted which does not comply with these terms. 